\title{
People with traumatic brain injuries used various strategies to deal with labels applied by society
}

\author{
Nochi M. Struggling with the labeled self: people with traumatic brain injuries in social settings. Qual Health Res 1998 Sep;8:665-81.
}

\section{Question}

How do interactions with society affect the sense of self of individuals with traumatic brain injuries (TBIs)?

\section{Design}

Grounded theory.

\section{Setting}

Midsized city in northeastern USA.

\section{Participants}

10 adults (age $24-54$ y, 8 men) with TBI (primarily as a result of car accidents) were recruited from a TBI support group. All participants lived in the community, had the skills to participate in interviews, and understood that they had TBI.

\section{Methods}

Data were collected using $\geqslant 2$ semi-structured interviews. Additional data were obtained from $>100$ hours of participant observation and from email messages from 13 people (age 26-61 y, 8 women) posted to a TBI internet discussion group. Field notes documented participants' non-verbal behaviour, activities in natural settings, and the author's reflections as an observer. Data were analysed using constant comparison analysis. Findings were confirmed with participants and with other people with TBI.

\section{Main findings}

The main theme expressed by participants was that society attached both negative and positive images to them that often contradicted their own self image. Participants felt that negative diagnostic labels such as TBI or head injury were associated with an image of abnormality. Some felt that the term brain injury connoted craziness or stupidity and were afraid that society would identify them as mentally ill or retarded. They felt that the term disabled was associated with an image of powerlessness and a loss of ability. Participants coped with these negative images using 4 strategies. Some controlled information about the self by selectively presenting the "normal" parts of themselves. By distinguishing functional changes from the self, participants emphasised their ability to complete a job rather than the process by which they did it. If participants could not present themselves as normal, they sometimes modified the meaning of normality or focused on specific aspects of normality (eg, activities of daily living rather than employment status). Some participants replaced the term TBI with terms that focused on things they had in common with other people (eg, having problems rather than TBI because "everybody ... has problems").

Participants also felt that society attached positive labels that contradicted how they defined themselves. Normality as a label was applied because many of the functional changes arising from TBI (eg, memory loss) are not visible. As a result, family, friends, and healthcare providers discounted the seriousness of the functional changes. Participants did not like the label of normality because of the threat of other labels that could imply that the person had a pathologic personality rather than a medical problem. For example, if a person with TBI accepted a label of normality, others could attribute functional inadequacies to laziness, stupidity, lack of ability, or faking to get extra compensation. To avoid these positive labels, participants used the term TBI strategically to communicate the nature of their functional changes. Some stressed the concept "brain" to indicate the cause of their changes, whereas others stressed the words traumatic or injury to convey the range and seriousness of their changes or to emphasise the cause as an external force rather than a pathological one.

\section{Conclusions}

People with TBI felt that society attached both negative and positive labels to them that often contradicted their own self image. They used various strategies to manage these inappropriate images.

Source of funding: not stated.

For correspondence: contact information not available.

\section{Commentary}

The study by Nochi provides insight into the reactions of individuals with TBI to labels imposed by society that counter their self image. The author used rigorous data collection methods and adhered to procedures of grounded theory. Concurrent analysis of the data to provide further direction in the interviews and concept development, $>100$ hours of participant observation, $>1000$ pages of field notes, $\geqslant 2$ interviews with each of 10 primary participants, and comparison of responses with 13 email participants enhanced the rigour of the study. As a result, an inductively developed theory about the labels attached by society to people with TBI, various management strategies, and the images that individuals with TBI desire emerged.
People with TBI strive to exclude from their identity labels that contribute to powerlessness, images of abnormality, and the risk of other undesirable labels. The importance of labelling in nursing is evident in other patient populations. For example, the term "non-compliant" is commonly applied to individuals who do not participate in a prescribed healthcare plan. Their "non-compliant" behaviour is assumed to be because of such factors as lack of money, intelligence, and convenience. Powerlessness, however, has been shown to be a factor in health defeating behaviours. ${ }^{1}$ A perception of powerlessness has been attributed to people who are chronically ill. Specific nursing interventions have been shown to be effective in alleviating perceived powerlessness in this population. ${ }^{2}$ Nochi's study enhances the nurse's awareness of the inappropriate labelling experienced by individuals with TBI. This may prompt nurses to explore the issues of labelling and powerlessness with people with TBI.

Jan Foster, RN, CGRN, MSN Assistant Professor, College of Nursing Houston Baptist University Houston, Texas, USA

1 Gallagher SM. Powerlessness as a factor in health defeating behaviour. Ostomy Wound Manage 1997;43:34-8, 40, 42 .

2 Kubsch S, Wichowski HC. Restoring power through nursing intervention. Nurs Diagn 1997;8:7-15. 